\title{
Clinical Application of Single Dual-Energy Subtraction Technique With Digital Storage-Phosphor Radiography
}

\author{
Ryouichi Kamimura and Tsutomu Takashima
}

We used a single-exposure dual-energy subtraction technique with a storage-phosphor computed radiography (CR) system to evaluate various pulmonary diseases. Clinical applications of this method have been studies at our institution. In our experience, the dual-energy subtraction technique provided better visualization of pulmonary nodules, tracheobronchial abnormalities, pulmonary vascular diseases, calcification in a nodule, and rib lesions than conventional CR images did. Single-exposure dual-energy subtraction images were obtained with a sandwich technique using two storage-phosphor imaging plates with a 1-mm-thick copper filter interleaved between the two. The front plate obtains a lower kilovoltage image, whereas the back plate obtains a higher kilovoltage image. We can visualize soft tissue and bone images as well as unsubtracted digital images by the subtraction process.

Copyright $\odot 1995$ by W.B. Saunders Company

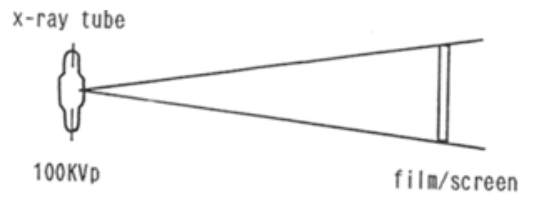

phototimer

Dual-energy subtraction radiography

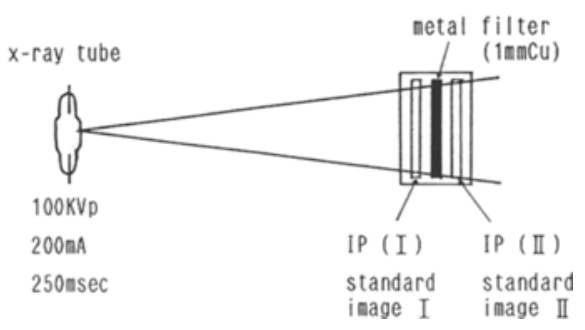

Fig 1. (A) Imaging techniques and radiation exposure. (B) Dual-energy subtraction bone and soft-tissue images.
KEY WORDS: Computed radiography, single-exposure dual-energy subtraction, Dual-energy subtraction image, chest radiography.

D IAGNOSTIC ACCURACY in detecting pulmonary nodules was compared among conventional film-screen radiography (CFS), plain storage-phosphor radiography, and dualenergy subtraction soft-tissue image (Fig 1).

From the Department of Radiology, Kanazawa University School of Medicine, Ishikawa, Japan.

Address reprint requests to Ryouichi Kamimura, MD, Department of Radiology, Kanazawa University School of Medicine, 13-1, Takaramachi, Kanazawa city, Ishikawa 920, Japan.

Copyright $(1995$ by W.B. Saunders Company

0897-1889/95/0801-1007\$3.0010
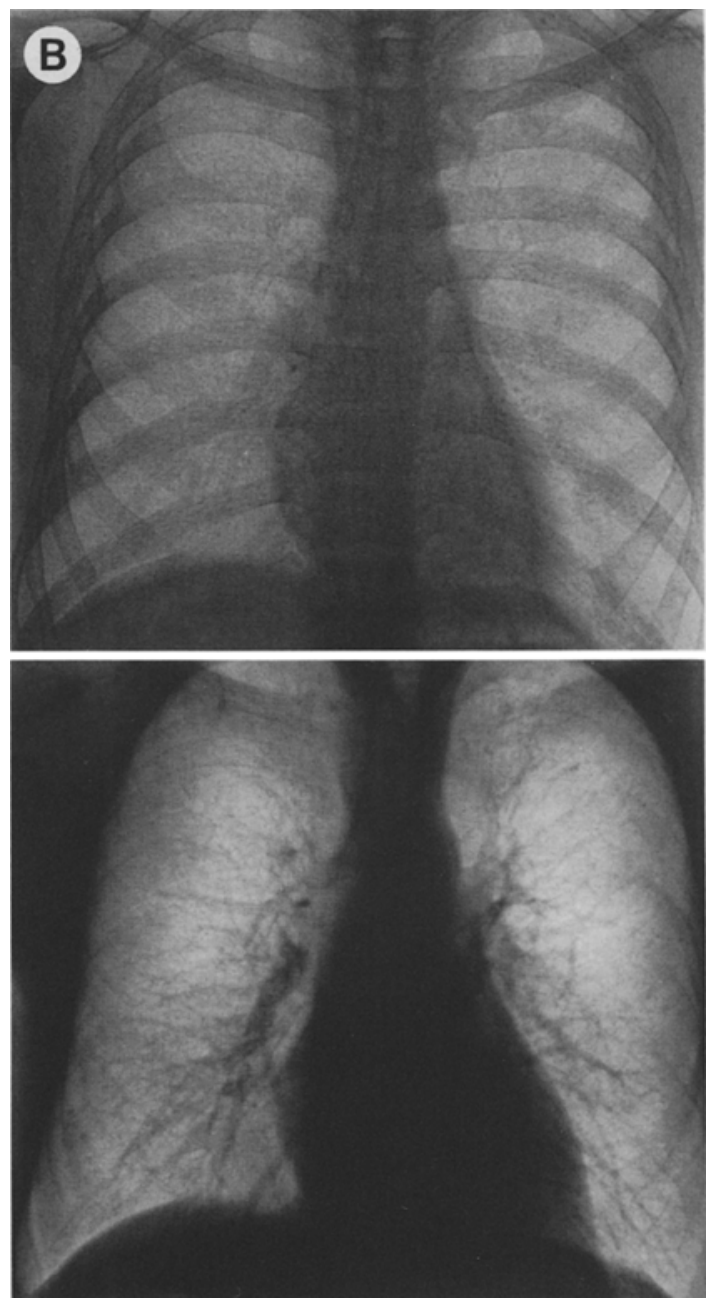
The study population consisted of 50 patients with a noncalcified pulmonary nodule $2 \mathrm{~cm}$ or less in diameter. The diagnosis of a solitary nodule was confirmed by surgery, and most were primary lung cancer nodules. Fifty control patients without evidence of pulmonary nodules were confirmed by computed tomography (CT) scan or serial chest radiographs.

To compare diagnostic accuracy among CFS, plain digital, and subtraction soft-tissue image, these three image sets were presented to 12 readers. Seven were experienced radiologists and 5 were residents in our department. The readers used a five-level scale to rank their confidence $(1$, definitely negative; 2 , probably negative; 3 , indeterminate; 4 , probably positive; 5 , definitely positive). Detectability was judged by receiver-operating characteristic (ROC) analysis.

The average ROC area of CFS was 0.893 , the average ROC area of plain digital was 0.896 , and the average ROC area of subtraction soft tissue image was 0.950 (Fig 2). Dual-energy image was significantly superior to CFS and plain digital images in the detection of pulmonary nodules.

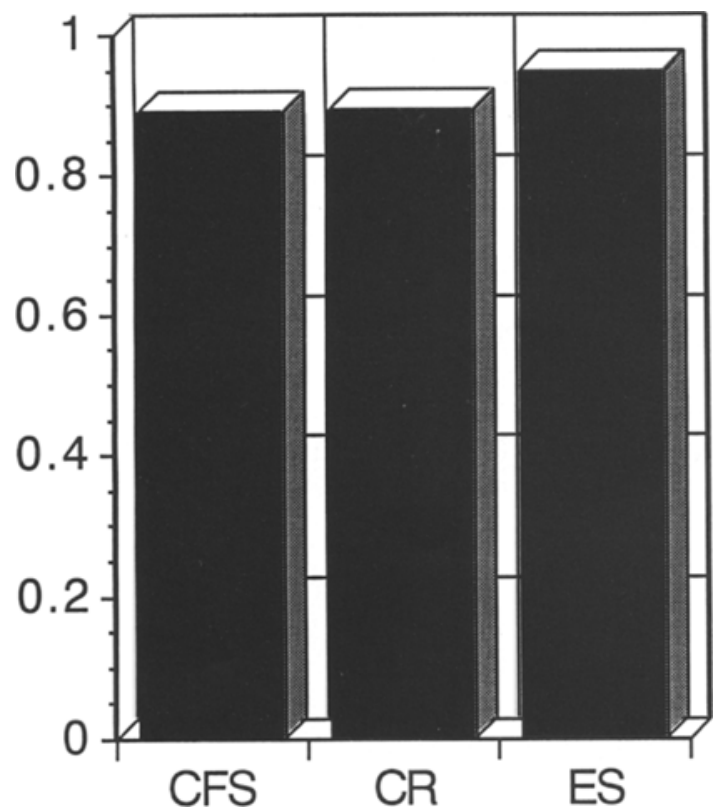

Fig 2. Clinical study on the detectability of pulmonary nodules. Areas under ROC curves for 12 observers on each modality. CFS, $0.893 \pm 0.014 ;$ CR, $0.896 \pm 0.031 ; \mathrm{ES}, 0.950 \pm$ 0.027 .

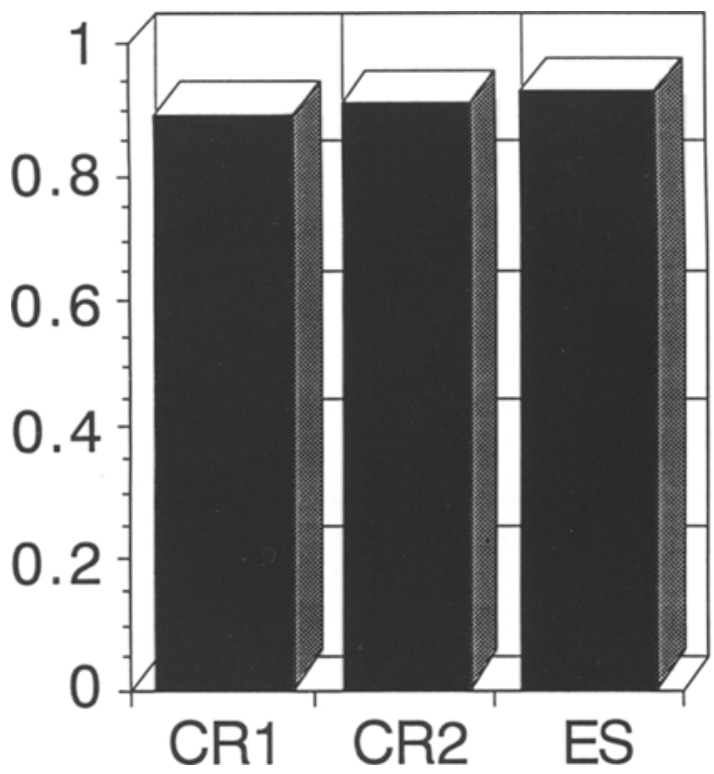

Fig 3. Screening for tracheobronchial diseases with digital storage-phosphor radiography. Areas under ROC curves for six observers on each image. Standard image (CR1), $0.896 \pm$ 0.038 ; enhanced image (CRZ), $0.910 \pm 0.051$; subtraction image (ES), $0.932 \pm 0.058$.

We conclude that dual-energy subtraction soft-tissue image improved the detection of pulmonary nodules by eliminating overlying bony structures.

\section{SCREENING FOR TRACHEOBRONCHIAL DISEASES WITH DIGITAL STORAGE-PHOSPHOR RADIOGRAPHY}

We investigated the clinical usefulness of dual-energy subtraction images in the screening of the tracheobronchial lesions. We compared the detectability of tracheobronchial abnormalities on the dual-energy soft-tissue images with that on plain unsubtracted digital images. Six observers viewed 40 images obtained in 20 normal subjects and 20 abnormal subjects. Tracheobronchial abnormalities were confirmed by bronchofiberscope. ROC analysis was also used.

The detection of tracheobronchial abnormalities on subtracted images was significantly better than that on plain digital images (Fig 3) Our results indicate that single-exposure dualenergy subtraction images improve the detection of tracheobronchial abnormalities in chest radiography. The major reason for this improvement was removal of the thoracic spine. 


\section{CLINICAL APPLICATION FOR DIAGNOSIS OF PULMONARY VASCULAR DISEASES}

To evaluate the quality of pulmonary vasculature images obtained by dual-energy techniques with storage phosphor radiography, we compared the image quality of pulmonary vasculature on the dual-energy subtraction images with that on plain digital images in 25 normal patients and 13 patients with pulmonary vascular abnormalities such as vascular malformations, or localized decrease of pulmonary blood flow caused by central lung cancer. The differences in the quality were assessed by three chest radiologists by direct comparison as follows: (1) energy-subtraction image (ES) is significantly better than plain computed radiography (CR) image; (2) ES is slightly better than CR; (3) ES is equal to $C R$; and (4) CR is significantly better than ES.

In visualization of the pulmonary vessels of normal patients, dual-energy soft-tissue images were equal or inferior to the original unsubtracted digital images probably because of increased noise (Fig 4A). In the detection of some pulmonary vascular diseases, especially localized decrease of pulmonary blood flow, subtracted images resulted in improved conspicuity of involved site (Fig 4B). We conclude that for

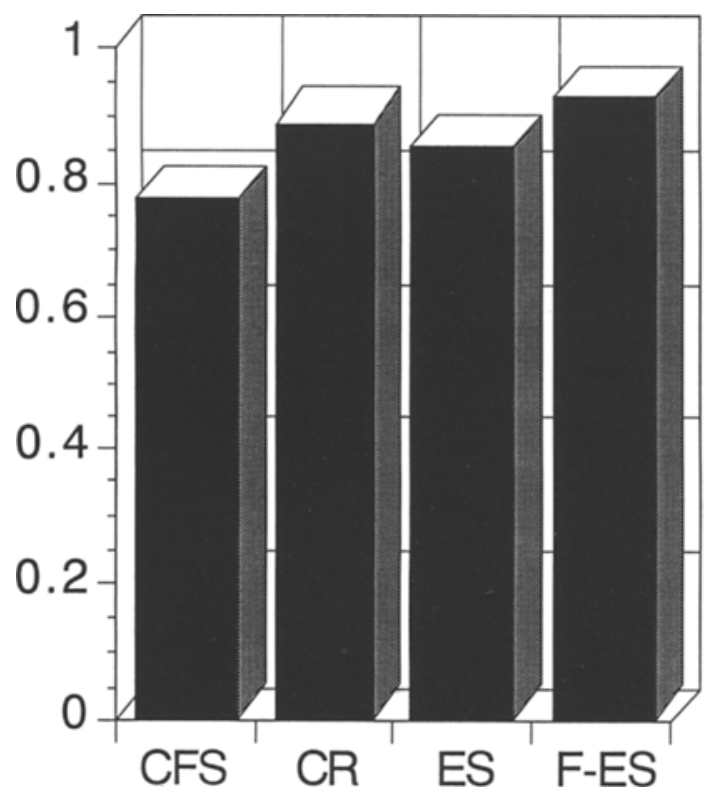

Fig 5. Calcification in putmonary nodules and detection with dual-energy digital radiography. Areas under ROC curves for nine observers on each image. CFS, 0.7748; CR, 0.8896; ES, 0.8529 ; Fine ES, 0.9235 .

visualizing the pulmonary vessels of normal patients, dual-energy were equal or inferior to the original unsubtracted digital images because of increased noise. However, in some

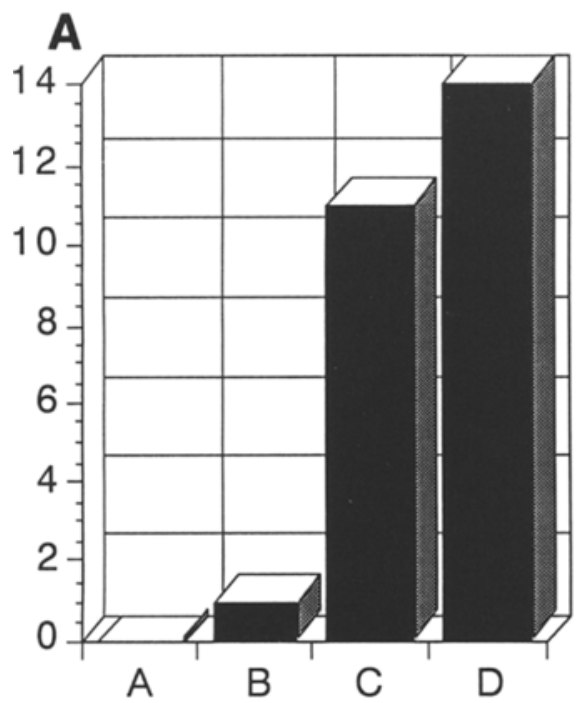

normal $(n=25)$

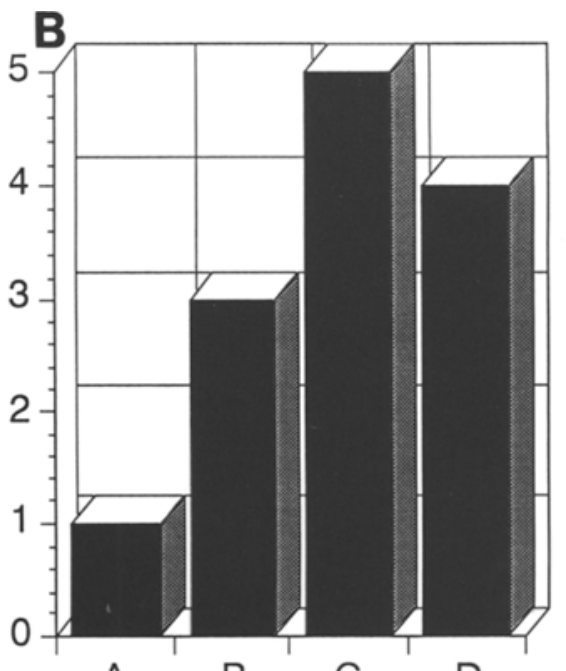

A B
C

pulmonary vascular diseases $(n=13)$

Fig 4. Clinical application for diagnosis of pulmonary vascular diseases. Comparison of image quality of pulmonary vessels in a normal patient (A); comparison of image quality of pulmonary vascular diseases. 
pulmonary vascular diseases, subtraction images resulted in improved conspicuity of involved sites compared with that of unsubtracted images.

\section{CALCIFICATION IN PULMONARY NODULES: DETECTION WITH DUAL-ENERGY DIGITAL RADIOGRAPHY}

We compared the diagnostic accuracy of detection of calcification in pulmonary nodules among CFS, plain digital, and dual-energy subtraction bone image. Twenty-one subjects with calcification in pulmonary nodules confirmed by CT scan and 21 control subjects without calcification in pulmonary nodules were selected. Nine observers were asked to detect calcifica- tion in the nodule using a five-level scale of confidence. The detection of calcification in a nodule with dual-energy bone image processed with noise-reduction algorithms was significantly better than that on other images (Fig 5). Dual-energy subtraction bone image processed with noise reduction algorithms provides useful information about calcification in a pulmonary nodule.

\section{CONCLUSION}

Clinical results from dual-energy imaging with a storage-phosphor CR system were promising. Dual-energy imaging provides better visualization of pulmonary nodules, tracheobronchial abnormalities, pulmonary vascular diseases, and calcification in a nodule. 